\title{
COAST - Customizable Online Syllable Enhancement in Texts: A flexible framework for automatically enhancing reading materials
}

\author{
Heiko Holz* (1) Zarah Weiß* ${ }^{*}$ Oliver Brehm Detmar Meurers \\ LEAD Graduate School \& Research Network \\ ICALL Research Group ${ }^{\dagger}$ \\ University of Tübingen \\ \{heiko.holz, zarah-leonie.weiss\}euni-tuebingen.de, \\ olibrehm@googlemail.com, dmesfs.uni-tuebingen.de
}

\begin{abstract}
This paper presents COAST, a web-based application to easily and automatically enhance syllable structure, word stress, and spacing in texts, that was designed in close collaboration with learning therapists to ensure its practical relevance. Such syllable-enhanced texts are commonly used in learning therapy or private tuition to promote the recognition of syllables in order to improve reading and writing skills.

In a state of the art solutions for automatic syllable enhancement, we put special emphasis on syllable stress and support specific marking of the primary syllable stress in words. Core features of our tool are i) a highly customizable text enhancement and template functionality, and ii) a novel crowd-sourcing mechanism that we employ to address the issue of data sparsity in language resources. We successfully tested COAST with real-life practitioners in a series of user tests validating the concept of our framework.
\end{abstract}

\section{Introduction}

Reading and writing disabilities are a pressing issue for today's society - approximately $4-8 \%$ of the German population suffer from dyslexia (Moll and Landerl, 2009; Bundesverband Legasthenie und Dyskalkulie e.V, 2014). Research on reading acquisition has shown that phonological awareness is a crucial skill for successful reading and writing acquisition (Röber-Siekmeyer, 2005). Important dimensions of phonological awareness are syllable synthesis and analysis. Syllable synthesis refers to the ability to blend syllables to a whole word, and syllable analysis to the ability of segmenting a word into its syllables. Experimental studies have shown that syllable synthesis and syllable analysis are essential components of evidence-based reading training (Galuschka and Schulte-Körne, 2016;

\footnotetext{
* These authors contributed equally to this work

† http://icall-research.de
}

Galuschka et al., 2014). Scheerer-Neumann (1981) have shown that specific training of segmenting words into syllables can improve reading accuracy of impaired German primary-school children significantly. Additionally, computer-based programs for primary-school children that sequentially speak and highlight syllables can facilitate the learning process of reading (Jiménez et al., 2007; Olson and Wise, 1992).

Based on these empirical findings, enhanced texts with custom spacing and syllables alternately displayed in different font colors are commonly used in teaching and learning therapy to support acquisition of reading and writing. This so-called Silbenmethode (syllable method) (Mildenberer Verlag, 2018) teaches children to focus on and understand syllables and their structures rather than single characters and is commonly used in Germany, which is reflected by popular reading materials, such as $A B C$ der Tiere and Leselöwe, and by available tools that facilitate the learning process of reading and writing, such as Celeco Druckstation and ABC Silbengenerator.

While first language acquisition happens through mere exposure, learning to read and write is a learned skill and thus requires explicit instruction, similar to Second Language Acquisition (SLA). In this regard, insights form SLA research on input enhancement relate to reading and writing acquisition. The well-established Noticing Hypothesis (Schmidt, 1990) states that learning requires the exposure to salient linguistic constructions that may be recognized by the learner. To facilitate this recognition of relevant linguistic constructions, Input Enhancement (Smith, 1993) has been successfully used, in particular in terms of visual enhancement of texts (e.g. colors, font changes, capitalization, spacing), cf. (Rello and Baeza-Yates, 2017; Zorzi et al., 2012; Meurers et al., 2010).

In response to this, we developed COAST. ${ }^{1}$

\footnotetext{
${ }^{1}$ www.sfs.uni-tuebingen.de/coast/
} 
COAST is a web-based application to easily and automatically enhance syllable structure, word stress, and spacing in texts. Its primary focus is on functionality and practicability. In terms of functionality, COAST offers a high degree of customization for text enhancement, supports management of annotation schemes, and includes syllable stress. The performance of detecting syllable stress strongly predicts dyslexia (e.g., Goswami et al. (2013); Landerl (2003)) and correlates highly with reading and writing skills (Sauter et al., 2012) and, thus, is of special importance for dyslexic children. Trainings to improve the awareness of syllable stress are being developed and evaluated (Holz et al., 2017). We extend the approach of text enhancement that are provided by state of the art tools to make syllable structures and stress more salient for German native (dyslexic) speakers using NLP resources. Enhancing the text with such additional linguistic information might boost children's ability to segment words into relevant components and might help them to learn to focus on relevant areas of words - as major orthographic challenges, such as vowel length markers, mainly occur in (conjunction with) stressed syllables (Staffeldt, 2010). To account for practicability, we implement this functionality by collaborating closely with prospective users and in particular teaching practitioners to meet real-life demands.

The remainder of the article is structured as follows: In Section 2, we report findings of a requirement analysis that we conducted in form of expert interviews prior to the system design to determine the wishes and needs of practitioners and compare COAST to two state of the art tools currently used in learning therapy and reading and writing acquisition. In Section 3, we describe the framework of COAST and explain the two core functionalities crowd-sourcing and text enhancement with real-life use cases. In Section 4, we evaluate the usability and user experience of COAST by means of user tests conducted with learning therapists and validate its practical applicability. We conclude by describing the current state of COAST and providing an outlook for its further development in Section 5.

\section{Requirements Analysis}

\subsection{Expert Interviews}

As the primary focus of our work was on the design of a tool that allowed for the immediate practical application by language teachers and learning therapists, we performed a requirement analysis for our system preceding its implementation. We conducted four expert interviews with teaching therapists to establish their wishes and requirements for a text-enhancement tool that would facilitate their work. During this process, we identified a series of concrete requests going beyond the tool's basic text analysis functionality. They were centered around four main issues: i) input/output options, ii) flexible customization settings, iii) user profiles and re-usability of settings, and iv) optional expert/user judgments.

Input/Output Options proved to be of particular interest for prospective users. They emphasized the wish to not only be allowed to upload their own texts, but also to be able to flexibly edit them while seeing the syllable enhancement. Therefore, we provide a text box for users in which they may enter and alter their texts. Regarding output options users expressed interest in being able to choose between the formats HTML, MS Word, and PDF/printing, or simply copying texts with enhancements to the clipboard. All of these were incorporated into our system.

Flexible Customization Settings were, aside from the I/O options, one of the most prominent user concerns. We found that the text representations should be customizable not only in terms of the basic text layout, but also preferably in all aspects of the actual syllable enhancement. Thus, users may freely customize the spacing of lines, words, syllables, and characters, as well as different font sizes. Furthermore, the visual syllable enhancement is customizable in terms of the colors used for stressed and unstressed syllables with the additional options to assign a separate color to secondary unstressed syllables. Colors may either be applied to the background or the font. Users may further decide to additionally highlight stressed syllables with bold font. They may also choose to mark syllable boundaries with a freely selectable delimiter. Finally, users can select certain partsof-speech to be either i) annotated, ii) marked as unstressed, or iii) ignored. Combined, these parameters allow for a highly customizable text design and visual enhancement, that gives users a high degree of freedom regarding the representation of their texts. 
User Profiles and Re-Usability became relevant concerns in the course of our expert interviews: Our flexible customization options give users the freedom to design text representations and visual enhancements that are tailored specifically to their purposes. However, users stressed the importance that they could re-use their elaborate customization across sessions, and that they need to be able to switch between various customized enhancement templates. To allow users to save, manage, and re-use their templates, we created user accounts that allow users to locally save their customization. Users may also save the texts they uploaded under a user-defined title in previous sessions.

Expert/User Judgments proved to be a final, pressing issue for prospective users: The option to adjust the automatic analyses in cases where users disagree with the syllabification or stress annotation performed by the system was crucial to our prospective users. To give them complete authority over their analyses, each of both analyses may be altered by the user on click. Furthermore, they asked us to flag words that were unknown to our system and thus more error prone. To facilitate manual corrections, we offer users to review all unknown words consecutively in a separate view, where they are supported by the syllabification and stress suggestions of our systems. All changes conducted by users are saved in their local syllabification data base and used for future analyses. Users may review and edit these new entries in their account settings. A final suggestion of our expert users was to allow the system to learn from user feedback. We thus include a crowd-sourcing based mechanism for updates to the global data base, which is explained in detail in Section 3.2.

\subsection{Related Work}

There are two dominant syllable enhancement tools for German whose functionality is centered around the so called Silbenmethode ("syllable method"), in which reading is taught by focusing on syllables and their pronunciation rather than single characters: the Silbengenerator ("syllable generator") and the Celeco Druckstation ("Celeco printing station"). ${ }^{2}$ Table 1 shows a comparison of the tools with COAST based on the characteristics that we identified in our expert interviews and some more

\footnotetext{
${ }^{2}$ We are not aware of any tools for the English market that provide any syllable enhancement beyond character-based markings.
}

\begin{tabular}{rccc}
\hline System Feature & Silbengenerator & Celeco & COAST \\
\hline Platform Independent & $x$ & $x$ & $\checkmark$ \\
Web-Based & $x$ & $x$ & $\checkmark$ \\
Freely Available & $(\checkmark)$ & $x$ & $\checkmark$ \\
Free Text Input & $\checkmark$ & $\checkmark$ & $\checkmark$ \\
Text Box & $x$ & $\checkmark$ & $\checkmark$ \\
Basic Text Layout Customization & $\checkmark$ & $\checkmark$ & $\checkmark$ \\
Additional Text Layout Customization & $x$ & $(\checkmark)$ & $\checkmark$ \\
Customizable Syllable Enhancement & $x$ & $\checkmark$ & $\checkmark$ \\
Configuration Templates & $x$ & n.a. & $\checkmark$ \\
Stress Annotation & $x$ & $x$ & $\checkmark$ \\
Syllable Arcs & $x$ & $\checkmark$ & $x$ \\
Customizable Analysis & $(\checkmark)$ & $(\checkmark)$ & $\checkmark$ \\
Crowd-Sourcing & $x$ & $x$ & $\checkmark$ \\
Exercise Generation & $\checkmark$ & $\checkmark$ & $x$ \\
\hline
\end{tabular}

Table 1: Comparison of $A B C$ Silbengenerator, Celeco Druckstation, and COAST.

general usability considerations.

Silbengenerator is a Windows program published by Mildenberger Verlag (2018). ${ }^{3}$ It is part of their $A B C$ der Tiere ("animal alphabet") series of learning materials based on syllabification as reading aid. Its main functionality is to allow teachers to visually enhance syllables in their reading materials. While the full version has to be purchased, a free demo is freely available for downloads on their web page.The tool allows users to upload own texts for analyses, but not to modify them from within the tool via some form of text box. Supported output formats for enhanced texts are MS Word or PDF/print. The general text layout is adjustable in terms of line spacing, fonts, font sizes, text alignment, line breaks, and background color. However, more advanced changes to the text layout, such as customized syllable, character, or word distances are not supported. The latest customized layout may be re-used upon system restart, but it is not possible to store multiple templates. Syllables are visually enhanced using the conventions of the $A B C$ der Tiere materials, which hyphenates syllables and additionally marks alternating syllables with red and blue font. Monosyllabic words default to blue. Word stress is not encoded. To accommodate limited printing capacities, syllables may be enhanced using gray and black instead of red and blue, but further customization is not supported. Users may locally overwrite the syllable boundaries set by the system for individual words by editing a plain text file outside of the program. Changes are applied to all documents upon restart. Changes during run-time or for individual documents are not supported User corrections are not

\footnotetext{
${ }^{3}$ For details, see: www. abc-der-tiere. de/index. php? id=388
} 
re-used to improve the system's syllabification performance. The Silbengenerator also includes a limited tutoring functionality, which includes two variations of syllable reading exercises as well as capitalization, vowel, and spelling training.

Celeco Druckstation is a Windows program distributed by Celeco (Klische, 2007). ${ }^{4}$ Since there is no free demo version, we base our review on the elaborate tool description provided on their web page. It should be pointed out that - unlike the Silbengenerator and our system - the Celeco Druckstation is distributed as a full fledged diagnosis and therapy tool for reading disorders for learning therapists and home tutoring alike. It thus provides a number of tests and exercises for reading and diagnosis, which are generated from texts specified by the user. This also includes a syllable enhancement facility that allows to load texts into the program, visually enhance syllables, and print them. Celeco Druckstation offers to adjust the basic text layout in terms of fonts, font size, font color, and background color. It also supports advanced layout modifications in terms of text segmentation: users may choose to put spaces after every syllable or every 3rd, 4th, or 5th character. Syllables are enhanced with two alternating, freely customizable colors, or with syllable arcs. No special encoding of word stress is offered. Users may provide individual syllable analyses of unknown words. These are saved in a local data base. However, the syllabification of known words can - as far as we could determine - not be altered by the user. We could not determine whether enhancement settings may be saved and re-used as templates.

\section{Tool}

\subsection{System Description}

We developed COAST as a platform-independent web-based tool that is deployed with Apache on a server hosted on the Amazon Web Services (AWS). ${ }^{5}$ The front-end was developed with HTML, CSS, JavaScript, and AngularDart. ${ }^{6}$ The backend was developed with Python using the frameworks Flask, ${ }^{7}$ and SQLAlchemy. ${ }^{8}$ We use spaCy (Honnibal and Johnson, 2015) for natural language processing (NLP).

\footnotetext{
${ }^{4}$ www . celeco.de/

5 WwW . aws . amazon. com/

${ }^{6}$ www . angulardart. org/

${ }^{7}$ WWw. flask.pocoo.org/

8 wWw. sqlal chemy. org/
}

Target Users are on the one hand teaching practitioners, but on the other hand any person with an interest in syllabified reading material, such as tutors or parents. We account for this divide with two separate types of user accounts: regular and expert users. Currently, this distinction is relevant for our crowd-sourcing mechanism, which is discussed in Section 3.2.

Analyzing Input Texts is the core functionality of COAST. Figure 1 shows the workflow of automatic text analysis and enhancement. Before users can enhance texts in the front-end as described in Section 3.2, texts need to be processed accordingly: First, spaCy is used for parsing, tokenization, and part-of-speech (PoS) tagging. The letters of a word and its PoS are used as a combined primary key to query the global and local database stored in SQLite. The global database is initialized with the German version of the language corpus CELEX2 of Baayen et al. (1995) and is available to all users. For the approximately 360,000 lemmas and inflected word forms that are included in CELEX2, we infer primary word stress and syllable structure from CELEX2's orthographic and phonetic transcriptions. The local database consists of manually annotated entries and is only available to the specified user. If an entry was found, the syllabification, syllable stress, and lemma of the word are returned. If no entry was found, the word is marked as unknown and must be manually annotated. Manually annotated entries are automatically stored in the local database of the user and forwarded to the crowd-sourcing mechanism explained in detail in Section 3.2. The annotated information is used afterwards to enhance syllables and words of the text as can be seen in Appendix A, Figure 5. Further linguistic information for each enhanced word may be obtained individually, see Appendix A, Figure 6.

\subsection{Features}

Crowd-Sourcing is one of COAST's most innovative features. We exploit the crowd-knowledge for long-term improvements of our automatic syllabification and word stress analysis. Currently, the crowd is derived from COAST's active users. To reliably identify not only syllable boundaries but also stress patterns is one of the biggest challenges in automatic syllable enhancement due to limitations of the available linguistic resources. This is especially true for languages other than English and 


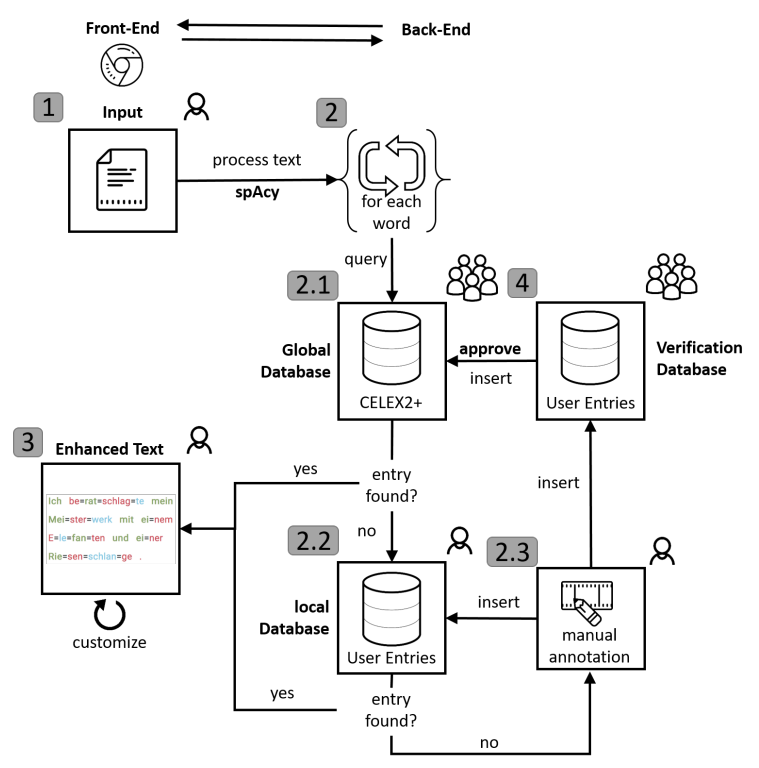

Figure 1: System overview of COAST.

for German this issue is particularly pressing, because irrespective of the size of the underlying data base, morphological composition and derivation are highly productive in German, which makes the occurrence of unknown words more likely. This issue of data sparsity is well-known from other NLP applications such as machine translation or information retrieval, and often addressed in work on compound splitting (Ziering and van der Plas, 2016; Weller et al., 2014). Furthermore, both processes may alter the word stress making the issue more difficult (Féry, 1998).

During the expert interviews it became apparent that prospective users prefer to be alerted to unknown words that may have been mis-analyzed, so they may review and if necessary manually correct them. In this context, we found that users would also prefer the system to learn from their corrections so that they could contribute to making the system more efficient in the long run. Together with our experts we therefore developed a crowdsourcing mechanism, that would allow local corrections of users to be incorporated to our system's global data base after they have been verified by either two more users or an expert user. We derived this role of an expert user to prioritize the votes of learning therapists and linguists over layman judgments. Upon registration, new users may self-identify as experts or as regular users. A verification of this self-assignment remains for future work.Experts may also revoke crowd-induced updates to the data base. With this combined expertise and additional layer of control through experts, our system may draw from a constantly growing pool of analyses, which ultimately increases its usability and robustness while building a promising resource for future work.

The verification mechanism is located on a separate page that asks users to voluntarily identify syllable boundaries and word stress of words unknown to the system. To facilitate analyses, we provide users with information on how our automatic tools would analyze a word as help as well as with previous analyses of other users. We incorporate the freely accessible MARY-TTS (Schröder and Trouvain, 2003) for automatic suggestions for stress annotation and Pyphen for syllabification. We require users to manually annotate each word unknown to the database due to insufficient performance of automatic stress assignment. This is an extensible framework, which may be expanded with more detailed information in the future.

The following use case illustrates this process: User 1 uploads a text containing two words unknown to the system: Hitzeschock ("heat shock") and Hacken ("heels"). She is asked to determine the syllable boundaries and stress (marked in bold font) for both words and submits Hit-ze-schock and Hac-ken. This syllabification assumes a bisyllabic consonant doubling for both terms. While this is correct for most consonants at syllable boundaries, $c k$ is an exception to this rule which is unknown to many laymen. Thus, when our system prompts Users 2 and 3 to verify User 1's analysis, they agree with her and both analyses are updated to the global data base. User 4 uploads another text containing Hitzeschock. Afterwards, the word is not flagged as unknown, but analyzed together with all other words that were originally included in the data base. Expert User 5 is asked to review the updates to the data base. She identifies the mistake that has been made and revokes the analysis of Hacken to $\boldsymbol{H a}$ cken. The entry is immediately corrected in the global data base and will be displayed correctly for all future analyses.

Text Enhancement is the core functionality of our tool. We enhance syllable boundaries as well as - unlike other systems - stress. For this, we rely on automatic analyses and manual post-hoc corrections by the user for words that are flagged as unknown: Our expert interviews clearly showed that prospective users not only prefer a high degree of customization in the visual representation 


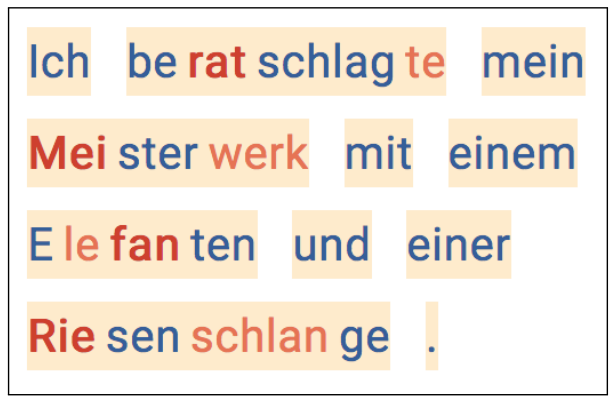

Figure 2: Template inspired by $A B C$ der Tiere.

of their texts, but also want to re-use and switch between templates. Therefore, we not only facilitate advanced customization options for the text and enhancement layout, but also allow users to store various templates, which may be consecutively applied to a text with a simple click.

Our settings feature two main categories: First, they allow to modify the enhancement of syllable stress by allowing users to choose freely the colors assigned to i) stressed syllables, ii) unstressed syllables, and optional iii) the second unstressed syllable. Stressed syllables may be enhanced with bold font. Users can choose whether to apply the color enhancement to the font or the syllables' background. Furthermore, syllable boundaries may be made more salient by using a syllable delimiter character that users may choose freely. Finally, users may specify to which extend certain parts of speech should be analyzed, e.g. they may choose to ignore articles or to default connectives to be enhanced as unstressed. Second, they allow users to customize the text layout independent of the syllable enhancement. This includes basic options such as adjusting font size or line space. However, we also allow to freely choose the distance of words, syllables, and characters. Users may further make word boundaries more salient by choosing a background color for them. The combination of these syllable enhancement and text layout settings may be saved under a descriptive title as a template, which may be re-used and altered at any point across texts or sessions.

The following use case illustrates how this works: User 1 works with children with reading disabilities from two groups: Group A uses the $A B C$ der Tiere materials in school. The children are thus used to the blue and red layout, which User 1 wants to alter as little as possible, while still providing her pupils with materials that also mark syllable stress. Therefore, she customizes a template to use the

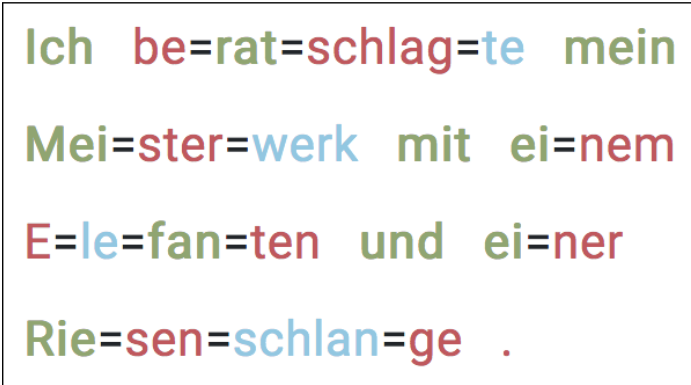

Figure 3: Template inspired by Leselöwen.

$A B C$ der Tiere style for her enhancement. Figure 2 shows the result for the sentence Ich beratschlagte mein Meisterwerk mit einem Elefanten und einer Riesenschlange ("I consulted my masterpiece with an elephant and a giant snake").

She sets the marking color of stressed syllables to dark red and of unstressed to blue. In order to make the alternation of syllables more salient, secondary unstressed syllables are also marked in red. To clearly distinguish them from stressed syllables, she additionally uses bold font to mark stress and uses a lighter type of red to mark secondary unstressed syllables. Because $A B C$ der Tiere colors monosyllabic words in blue, User 1 further sets typically monosyllabic parts of speech, such as articles and prepositions, from the analysis to be analyzed as unstressed. Finally, she makes syllable boundaries more salient by widening the distance between syllables. To make word boundaries more salient, despite this increased syllable distance, she further widens word distance and assigns a beige background color to words.

Children from Group B do not use the $A B C$ der Tiere materials at school, but they are reading syllabified stories at home from the Leselöwen ("reading lions") materials by the Loewe publisher." These materials use three colors to mark alternating syllables and they do not treat monosyllabic words differently from others. For this group, too, User 1 wants to make stressed syllables more salient in her materials, while otherwise not deviating much from the layout the children are already used to. Thus, she designs a second layout which mimics the Leselöwen style. The result of applying this template to the same sentence she used for Group A may be seen in Figure 3.

The colors used by Leselöwen are green, red, and blue. She assigns stressed syllables the color

\footnotetext{
${ }^{9}$ www. loewe-verlag.de/ content-1013-1013/leseloewen/
} 
green and again additionally marks them with bold font. Unstressed and secondary unstressed syllables are colored red and blue. Because this style already features three colors, she does not want to use a background color for words. At the same time, she wants to make word as well as syllable boundaries more salient. For this, User 1 chooses to mark syllable boundaries with a delimiter (in this case $=$ ) but without additional space between syllables and increases the distance between words. While the initial customization took a couple of minutes, User 1 may re-apply her two templates to any text in the future, reducing the time required for customization to mere seconds. She may also alter the templates at any time or add new ones when required.

\section{Evaluation}

We conducted user tests to evaluate COAST with both practitioners as well as with non-experts. Prior to these, we performed an internal pilot testing to identify runtime issues that are not directly related to the functionality of COAST.

Five scenarios were defined to evaluate the tool's functionality, usability, and user experience. They cover i) account creation, ii) text analysis and enhancement, iii) generation and use of annotation templates, iv) reuse of previously stored texts, and v) verification of user-generated entries ("crowdsourcing").

In the first scenario, the users were asked to create an account with given credentials.

The second scenario consisted of four major steps: First, users were asked to log into the recently created account. Secondly, they had to switch to the Text Analysis view of the tool and to analyze and enhance a given text. After analyzing the text, they were told to clarify all words unknown to the system, which are flagged and shaded in red. Finally, users were asked to adjust the annotation settings based on their personal preferences.

The third scenario covered the instructed generation and use of annotation templates. Users were asked to rebuild two annotation schemes by adjusting the annotation settings and save them as new templates.

In the fourth scenario, users were asked to store the analyzed text in their account and re-analyze it by selecting the stored text in the Account view of the tool.
In the fifth and final scenario, users were required to verify entries added by other users that are unknown to the global database. In order to do so, they were asked to switch to the Verification view (see Figure 7) and approve or edit five entries.

The second, third, and fifth scenario are of special importance as they cover the core-functionality of COAST and can be seen in Figure 8.

User Tests were conducted by seven users from two groups: three experts (learning therapists) to receive subject-specific feedback and four laymen to evaluate the general usability of the tool. The three experts were women aged between 40 and 51 $(M=45)$. The laymen aged between 22 and 27 $(M=25)$ included two men and two women with non-educational professions. The user test was carried out equally for both groups. None of the participants had interacted with the system before.

We used the after-scenario questionnaire (ASQ) by Lewis (1995) for quantitative data analysis. They were answered for each scenario directly after its completion. The ASQ consists of three questions covering ease of use, time efficiency, and documentation of the tool:

1. Overall, I am satisfied with the ease of use of completing the tasks in this scenario

2. Overall, I am satisfied with the amount of time it took to complete the tasks in this scenario

3. Overall, I am satisfied with the support and documentation when completing the tasks

We used a five-point Likert scale ranging from strongly agree to strongly disagree.

For qualitative analysis, the users were explicitly instructed to "think-aloud" (Rauterberg, 1996) while working on a scenario, thus told to accurately comment each of their actions and to express expectations, thoughts, and critics.

The user test was carried out as follows: Users were free to use their preferred browser for the user test. The default browser was Google Chrome. The user test was conducted on the users' personal laptop if possible, to recreate their home or work environment and to mimic a real-life application as close as possible. If no personal laptop was available, users were provided with one. All input devices were configured according to user preferences. After setting up the work place, users were informed and instructed about the procedure of the user test, its purpose and the think-aloud method. 
After clarifying all questions, users processed all scenarios consecutively in fixed order. The user tests were concluded with an interview to get general feedback and to assess the usefulness of the tool with respect to the users' professions.

\subsection{Results}

The results of the second, third, and fifth scenario are explained in detail due to their relevance, results for scenario one and four can be found in the Table 2.

We normalized the options of the ASQ to range from -2 (strongly disagree) to +2 (strongly agree) with 0 (neither) being neutral. In the following, we report positive values (i.e. agree, strongly agree) as positive feedback, negative values (i.e. disagree, strongly disagree) as negative feedback. For a more detailed differentiation of the user feedback, please see Figure 4.

The second scenario was successfully completed by all users. The ease of use $(M=1.57, S D=$ $0.53)$ and documentation $(M=1.57, S D=0.53)$ of the tool was rated $100 \%$ positively in the ASQ, time efficiency received $86 \%$ positive and $16 \%$ neutral ratings $(M=1.57, S D=0.78)$. Some users criticized the layout of the Text Analysis view, suggesting a more compact representation of the annotation settings.

The third scenario was completed by five users without help, two needed hints from the investigator to complete all tasks. While the first template could be rebuilt by all users, two users required help with the second template. Error source was the confusion about and between the features Silbe hervorheben (enhance syllables), with which either the fore- or background color of syllables could be adjusted accordingly, and Wort Hintergrundfarbe (word background color), with which the background color of words could be set (see Figure 5). Three users completed this scenario by trial-and-error. The ease of use of this scenario was

\begin{tabular}{lccccc}
\hline Question & \multicolumn{5}{c}{ Rating } \\
& $\mathbf{- 2}$ & $\mathbf{- 1}$ & $\mathbf{0}$ & $\mathbf{1}$ & $\mathbf{2}$ \\
\hline \multicolumn{5}{c}{ Scenario 1 } \\
ease of use & $0 \%$ & $0 \%$ & $0 \%$ & $29.0 \%$ & $71.0 \%$ \\
time efficiency & $0 \%$ & $0 \%$ & $0 \%$ & $0 \%$ & $100 \%$ \\
documentation & $0 \%$ & $0 \%$ & $0 \%$ & $29.0 \%$ & $71.0 \%$ \\
Scenario 4 & & & \\
ease of use & $0 \%$ & $0 \%$ & $0 \%$ & $28.6 \%$ & $71.4 \%$ \\
time efficiency & $0 \%$ & $0 \%$ & $0 \%$ & $28.6 \%$ & $71.4 \%$ \\
documentation & $0 \%$ & $0 \%$ & $0 \%$ & $28.6 \%$ & $71.4 \%$ \\
\hline
\end{tabular}

Table 2: Results of the ASQ for scenario 1 and 4.

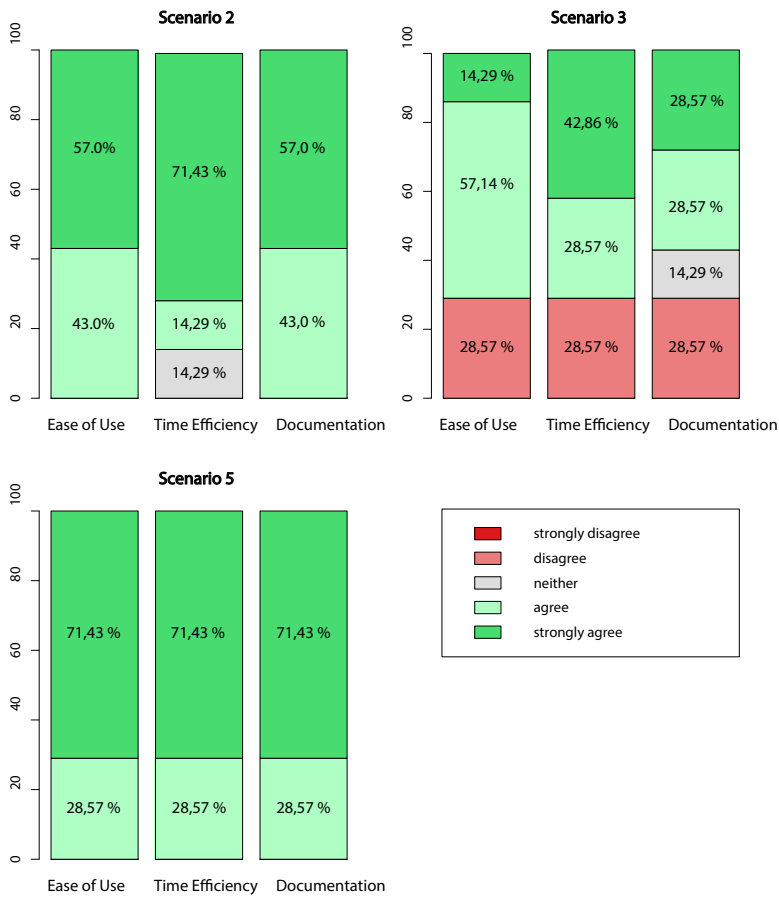

Figure 4: Results of the after-scenario questionnaire completed for scenario 2,3 , and 5 .

rated $71.4 \%$ positively and $28.57 \%$ negatively ( $M=0.57, S D=1.27)$, the time efficiency $71 \%$ positively and $29 \%$ negatively $(M=0.86, S D=$ 1.34 ), and $57.2 \%$ positively, $14.3 \%$ neutral, and $28.6 \%$ negatively in terms of documentation and support $(M=0.57, S D=1.14)$.

The fifth and last scenario was completed by all users successfully. Ease of use, time efficiency, and documentation of this functionality were rated $100 \%$ positively $(M=1.71, S D=0.49$ for each item respectively). Users suggested to design this functionality to be more user friendly by displaying and processing multiple entries at once.

The think-aloud and concluding interviews additionally revealed general layout and design flaws of COAST's visual appearance. While this goes beyond the scope of this paper, we list problems, comments, and feature requests directly linked to the core features of our tool: i) the general navigation of the tool was not very intuitive and selfexplaining, ii) some features could only be accessed with scrolling, which was not explicitly visible to the users, iii) some features, e.g. background color of words and syllable enhancement, need explicit documentation/tutorials, iv) some users asked for a simple solution to color syllables alternately independently of syllable stress, v) the feature to not enhance monosyllabic words instead 
of unchecking the annotation of typically monosyllabic parts of speech was requested, vi) fore- and background color of syllables and words should independently be customizable.

\section{Conclusion and Outlook}

COAST is a highly user-oriented, platform independent, web-based and easily extensible framework for the automatic augmentation of texts with syllable, stress, and word enhancement. It was developed in close collaboration with practitioners and includes a series of features which were explicitly requested by prospective users and that are lacking from currently available, state of the art systems. This paper presents and evaluates its ability to generate appropriate reading materials based on real-life use cases. Additionally, we evaluated the practical applicability of our tool by conducting user tests based on a series of real-life scenarios.

Our exemplary enhanced texts (see Figure 2 and 3 ) prove that appropriate reading materials can be easily generated automatically, customized, and exported with COAST. The use cases show that the tool meets the requirements deduced from the a priori requirement analysis based on our expert interviews. Compared to other tools that support syllable enhancement, COAST offers a higher degree of customization and more features, such as annotating syllable stress, setting spacing of lines, words, syllables, and characters. The automatic analysis of syllable stress and part of speech also make COAST linguistically more informed than other tools. Finally, we carried out user tests with special focus on practical application. These indicate that the majority of users were able to solve the tasks intuitively and time efficiently for each of the scenarios.

We have successfully shown that the current version of COAST allows practitioners to generate enhanced texts as reading materials for their teaching. Being able to save annotation templates and texts has proven to be an especially useful functionality to easily generate new reading materials within the application with little time effort. Furthermore, COAST features a novel crowd-sourcing approach to overcome the pressing issue of limited resources and data sparsity. This is particularly relevant for languages other than English. Currently, our tool illustrates this for the German language. However, the entire framework was designed to be easily extended for any other language for which sufficient resources are available.

Our consultation with prospective users also yielded a series of practical suggestions to optimize user experience further and to include more features. In particular, we aim at including the features discussed in Section 4.1. We also plan to redesign COAST's visual appearance. Furthermore, we intend to elaborate on the current documentation and to provide application-oriented feature tutorials. To improve the reliability of our proposed crowd-sourcing mechanism, we plan to address the verification of user roles, i.e. expert and regular users. In this regard, the need of further user type customization shall be analysed and implemented accordingly. Finally, we intend to carry out user studies to compare COAST's efficiency and efficacy to state of the art tools that support syllable enhancement in texts.

Our ultimate goal is to develop and include a front-end for learners, the COAST App. This results in a tutoring system offering reading and spelling exercises optimized for mobile devices. The current COAST Tool could be used by practitioners to generate teaching materials to be shared with the COAST App and, thus, to supply exercises directly to their pupils.

\section{Acknowledgments}

This research was funded by the LEAD Graduate School \& Research Network [GSC1028], a project of the Excellence Initiative of the German federal and state governments. Heiko Holz is a doctoral student at the LEAD Graduate School \& Research Network.

\section{References}

R. Harald Baayen, Richard Piepenbrock, and Léon Gulikers. 1995. CELEX2 LDC96L14. Web Download. Philadelphia: Linguistic Data Consortium.

Bundesverband Legasthenie und Dyskalkulie e.V. 2014. Der aktuelle Wissensstand über Legasthenie.

Caroline Féry. 1998. German word stress in optimality theory. Journal of Comparative Germanic Linguistics, 2(2):101-142.

Katharina Galuschka, Elena Ise, Kathrin Krick, and Gerd Schulte-Körne. 2014. Effectiveness of Treatment Approaches for Children and Adolescents with Reading Disabilities: A Meta-Analysis of Randomized Controlled Trials. PLoS ONE, 9(2):e89900.

Katharina Galuschka and Gerd Schulte-Körne. 2016. The Diagnosis and Treatment of Reading and/or 
Spelling Disorders in Children and Adolescents. Dtsch Arztebl Int., 113(16):279-86.

Usha Goswami, Natasha Mead, Tim Fosker, Martina Huss, Lisa Barnes, and Victoria Leong. 2013. Impaired perception of syllable stress in children with dyslexia: A longitudinal study. Journal of Memory and Language, 69(1):1-17.

Heiko Holz, Katharina Brandelik, Jochen Brandelik, Benedikt Beuttler, Alexandra Kirsch, and Detmar Meurers. 2017. Prosodiya-a mobile game for german dyslexic children. In International Conference on Games and Learning Alliance, volume 10653 of Lecture Notes in Computer Science, pages 73-82, Cham. Springer International Publishing.

Matthew Honnibal and Mark Johnson. 2015. An Improved Non-monotonic Transition System for Dependency Parsing. In Proceedings of the 2015 Conference on Empirical Methods in Natural Language Processing, pages 1373-1378, Lisbon, Portugal. Association for Computational Linguistics.

Juan E. Jiménez, Isabel Hernández-Valle, Gustave Ramírez, Ma Del Rosario Ortiz, Mercedes Rodrigo, Adelina Estévez, Isabel O'Shanahan, Eduarde García, and María De La Luz Trabaue. 2007. Computer speech-based remediation for reading disabilities: The size of spelling-to-sound unit in a transparent orthography. Spanish Journal of Psychology, 10(1):52-67.

Anja Klische. 2007. Leseschwächen gezielt beheben: Individuelle Diagnose und Therapie mit dem Programm celeco. Tectum-Verl.

Karin Landerl. 2003. Categorization of vowel length in German poor spellers: An orthographically relevant phonological distinction. Applied Psycholinguistics, 24(4):523-538.

James R. Lewis. 1995. IBM Computer Usability Satisfaction Questionnaires: Psychometric Evaluation and Instructions for Use. International Journal of Human-Computer Interaction, 7(1):57-78.

Detmar Meurers, Ramon Ziai, Luiz Amaral, Adriane Boyd, Aleksandar Dimitrov, Vanessa Metcalf, and Niels Ott. 2010. Enhancing authentic web pages for language learners. In Proceedings of the 5th Workshop on Innovative Use of NLP for Building Educational Applications (BEA-5) at NAACL-HLT 2010, pages 10-18, Los Angeles.

Mildenberer Verlag. 2018. Eine Einführung in die Silbenmethode. Mildenberer Verlag.

Mildenberger Verlag. 2018. ABC der Tiere - SilbenGenerator für die Grundschule.

Kristina Moll and Karin Landerl. 2009. Double Dissociation Between Reading and Spelling Deficits. Scientific Studies of Reading, 13(5):359-382.
Richard K. Olson and Barbara W. Wise. 1992. Reading on the computer with orthographic and speech feedback. Reading and Writing, 4(2):107-144.

Matthias Rauterberg. 1996. Usability Engineering. Morgan Kaufmann.

Luz Rello and Ricardo Baeza-Yates. 2017. How to present more readable text for people with dyslexia. Universal Access in the Information Society, 16(1):29-49.

Christa Röber-Siekmeyer. 2005. Die berücksichtigung des kindlichen sprachwissens für den schrifterwerb. In Hans-Werner Huneke, editor, Geschriebene Sprache. Strukturen, Erwerb, didaktische Modellbildungen, pages 129-144. Mattes Verlag, Heidelberg.

Katharina Sauter, Jürgen Heller, and Karin Landerl. 2012. Sprachrhythmus und Schriftspracherwerb. Lernen und Lernstörungen, 1(4):225-239.

Gerheid Scheerer-Neumann. 1981. The utilization of intraword structure in poor readers: Experimental evidence and a training program. Psychological Research, 43(2):155-178.

R. W. Schmidt. 1990. The Role of Consciousness in Second Language Learning1. Applied Linguistics, 11(2):129-158.

M. Schröder and J. Trouvain. 2003. The German Textto-Speech Synthesis System MARY: A Tool for Research, Development and Teaching. International Journal of Speech Technology, 6:365-377.

Michael Sharwood Smith. 1993. Input Enhancement in Instructed SLA. Studies in Second Language Acquisition, 15(02):165.

Sven Staffeldt. 2010. Einführung in die Phonetik, Phonologie und Graphematik des Deutschen Ein Leitfaden für den akademischen Unterricht. Stauffenburg, Tübingen.

Marion Weller, Fabienne Cap, Stefan Müller, Sabine Schulte im Walde, and Alexander Fraser. 2014. Distinguishing degrees of compositionality in compound splitting for statistical machine translation. In Proceedings of the 1st Workshop on Computational Approaches to Compound Analysis, pages 81-90.

Patrick Ziering and Lonneke van der Plas. 2016. Towards Unsupervised and Language-independent Compound Splitting using Inflectional Morphological Transformations. Proceedings of the 2016 Conference of the North American Chapter of the Association for Computational Linguistics: Human Language Technologies, pages 644-653.

Marco Zorzi, Chiara Barbiero, Andrea Facoetti, Isabella Lonciari, Marco Carrozzi, Marcella Montico, Laura Bravar, Florence George, Catherine PechGeorgel, Johannes C Ziegler, and Michael Posner. 2012. Extra-large letter spacing improves reading in dyslexia. Proceedings of the National Academy of Sciences, 109(28):11455-11459. 


\section{A Supplemental Material}

\section{A.1 Screenshots of COAST}

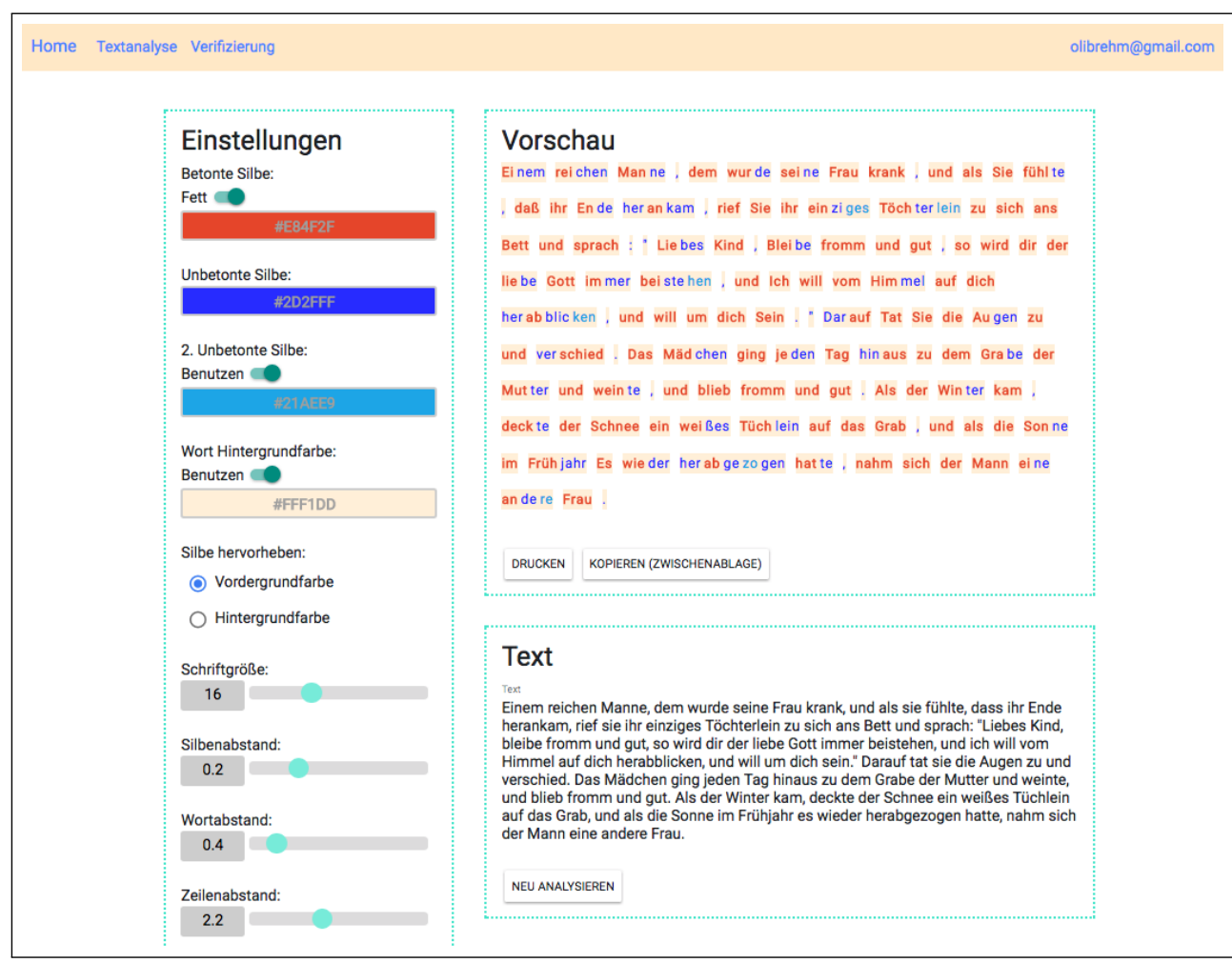

Figure 5: COAST - View for text analysis and enhancement. Users can insert or edit text in the lower text box. The preview of syllable enhancement is given in the upper box. On the left side, users can edit settings regarding syllable annotation: boldness, colors of stressed and unstressed syllables (either background or foreground), background color of words, font size, and spacing between syllables, words, and lines.

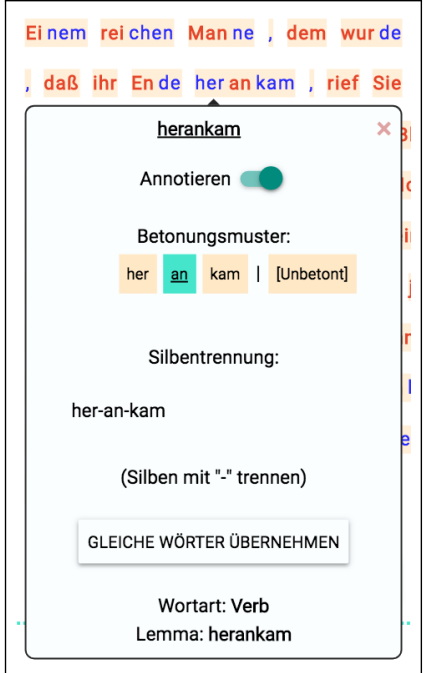

Figure 6: COAST - Word-Popup. Popup with additional information that is invoked when a known word is clicked in the text-view. At the moment, we offer information about syllabification, part of speech, and lemma. Users can additionally manually change the syllabification or stress assignment of the selected word and apply it to the preview. 


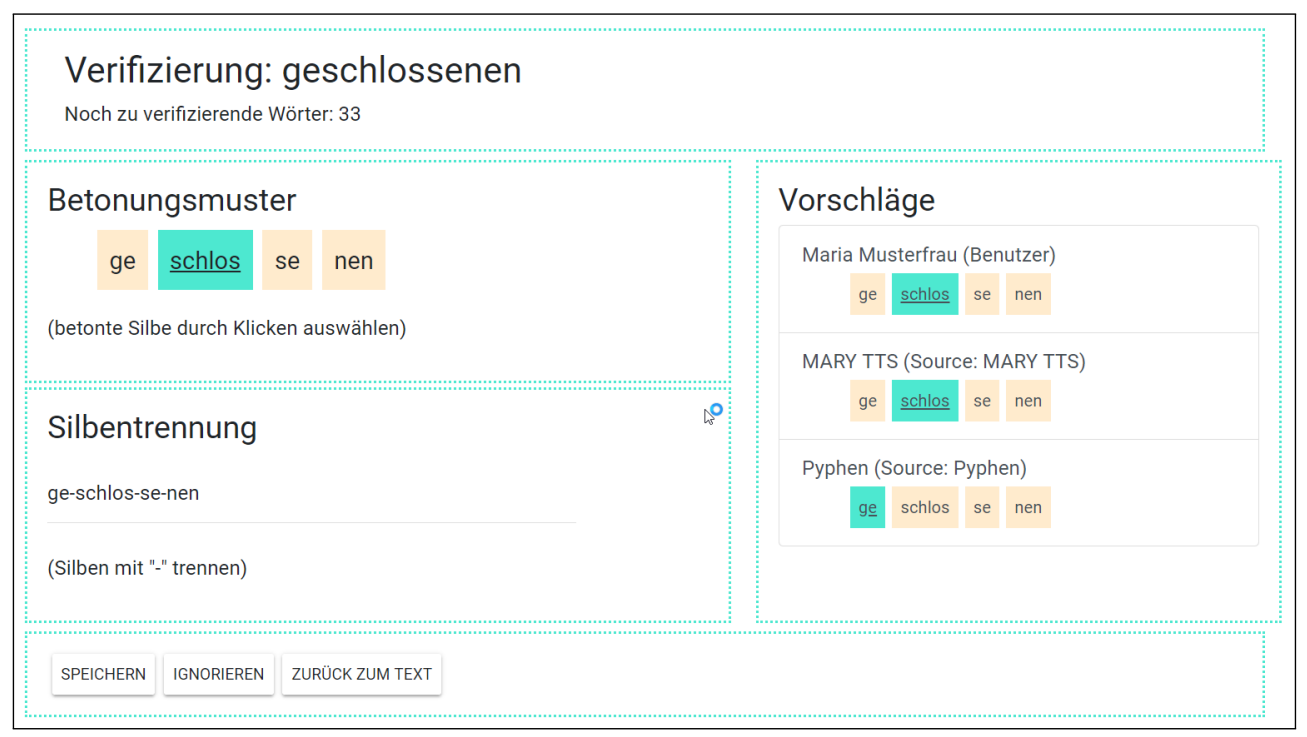

Figure 7: COAST - Verification-view of entries added by users unknown to the global database. Current word is geschlossenen (ge-schlos-sen-en, closed). Users can edit stress assignment and syllabification on the left side or agree to a user's judgment or to automatically generated suggestions on the right side.

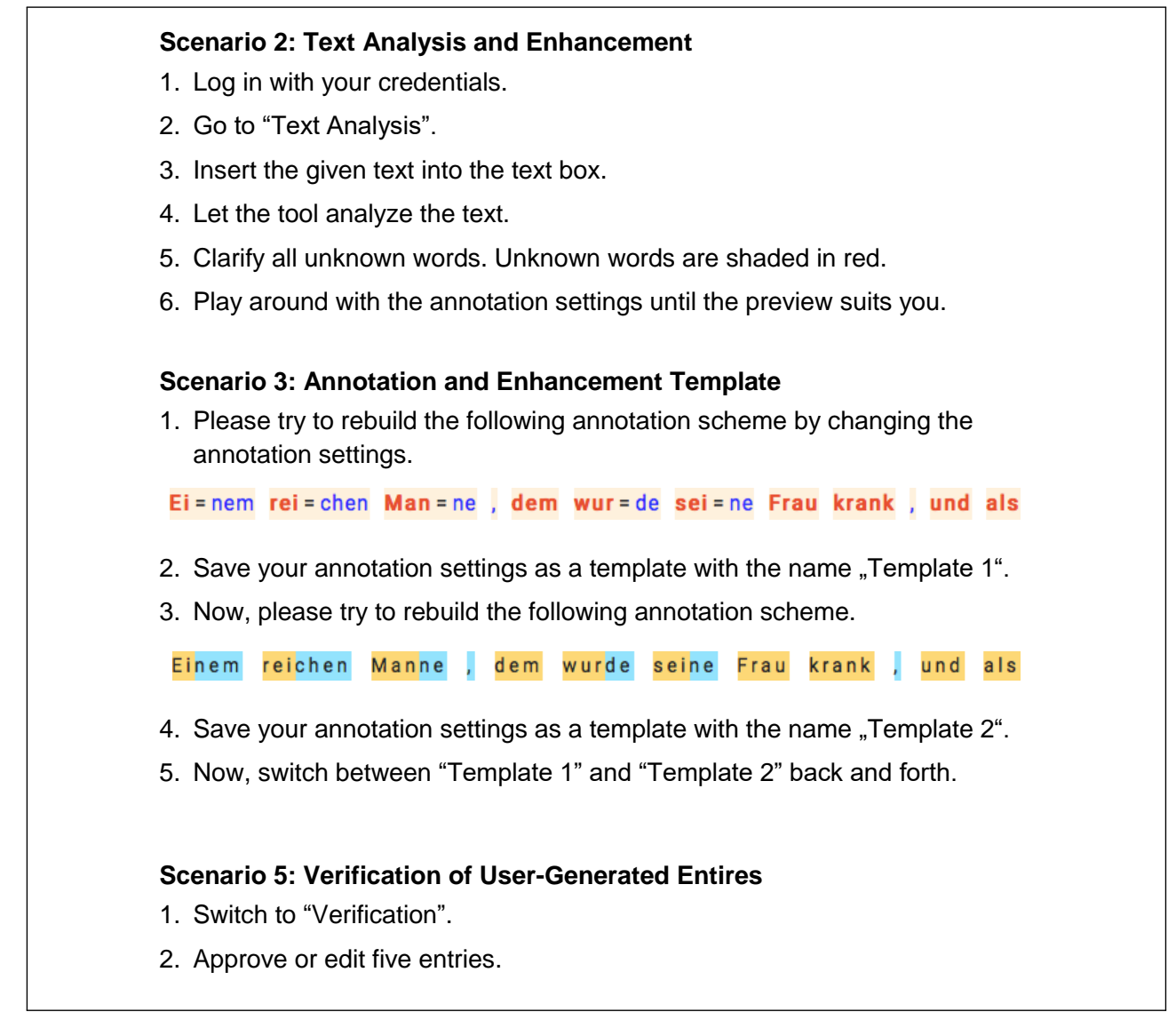

Figure 8: User tests of scenario 2 (text analysis and enhancement), 3 (creation and use of annotation and enhancement templates), and 5 (verification of user-generated entries). 